VOL. 53 (1996) [21-32]

\title{
GENERALISED HESSIAN, MAX FUNCTION AND WEAK CONVEXITY
}

\author{
X.Q. YANG
}

\begin{abstract}
In this paper, a second-order characterisation of $\eta$-convex $C^{1,1}$ functions is derived in a Hilbert space using a generalised second-order directional derivative. Using this result it is then shown that every $C^{1,1}$ function is locally weakly convex, that is, every $C^{1,1}$ real-valued function $f$ can be represented as $f(x)=h(x)-\eta\|x\|^{2}$ on a neighbourhood of $x$ where $h$ is a convex function and $\eta>0$. Moreover, a characterisation of the generalised second-order directional derivative for max functions is given.
\end{abstract}

\section{INTRODUCTION}

In this paper, characterisations of the generalised Hessian and the generalised second-order directional derivative introduced in [11] for certain max functions are obtained. It is shown how the twice weakly Gâteaux differentiability of max functions can be characterised. A necessary and sufficient condition for a real valued $C^{1,1}$ function to be $\eta$-convex is presented in a Hilbert space using the generalised second-order directional derivative. It is then shown that every $C^{1,1}$ function is locally weakly convex in a Hilbert space. This extends the corresponding results given in Hiriart-Urruty [3] and Vial [10].

Let $X$ be a Banach space. The class of $C^{1,1}$ functions is defined to be the set of all real valued continuously Gâteaux differentiable functions with locally Lipschitz gradients on $X$, denoted by $C^{1,1}(X)$. Consider the max function of the form $f(x)=[\max \{g(x), 0\}]^{2}$, where $x \in X$ and $g: X \rightarrow \mathbb{R}$. If $g$ is twice continuously differentiable, then it is known that $f$ is a $C^{1,1}$ function and various generalised Hessians for the function $f$ were given, for example, in Hiriart-Urruty, Strodiot and Nguyen [4] and Yang and Jeyakumar [11]. In this paper, we study the generalised Hessian introduced in [11] for $f$ when $g$ is a $C^{1,1}$ function. It is worth noting that squares of max functions appear in augmented Lagrangian function methods and smoothing

\section{Received 13th March, 1995}

This work was done while the author was a research student in Department of Applied Mathematics, The University of New South Wales. The author is grateful to Dr. V. Jeyakumar for his comments on several results of this paper.

Copyright Clearance Centre, Inc. Serial-fee code: 0004-9729/96 \$A2.00+0.00. 
approximation methods (see $[9,13,14]$ ). Thus generalised differentiabilities of max functions may be useful in studying optimisation methods.

In Hiriart-Urruty [3] and Vial [10], it was shown that in a finite dimensional space every $C^{1,1}$ function is locally weakly convex. This result is useful in establishing relations between $C^{1,1}$ functions and so-called lower- $C^{2}$ functions. We reprove this result in a Hilbert space by first obtaining a necessary and sufficient condition for $\boldsymbol{\eta}$-convex functions. This generalises a corresponding characterisation for convex $C^{1,1}$ functions in [11] to generalised convex functions and extends a result of finite dimensional spaces in $[\mathbf{1 0}]$ to infinite dimensional spaces.

\section{A Generalised Second-Order Directional Derivative}

Let $X^{*}$ be the dual space of $X$ and $\langle\cdot, \cdot\rangle$ be the canonical pair between $X^{*}$ and $X$. Let $g: X \longrightarrow \mathbb{R}$ be a locally Lipschitz function and $x \in X$. The Michel-Penot generalised directional derivative of $g$ at $x$ in the direction $u \in X$ is defined by

$$
g^{\circ}(x ; u)=\sup _{z \in X} \limsup \frac{g(x+s z+s u)-g(x+s z)}{s}
$$

and $g$ is said to be semi-regular at $x$ if the one-sided direction derivative

$$
g^{\prime}(x ; u)=\lim _{s \downarrow 0} \frac{g(x+s u)-g(x)}{s}
$$

exists and is equal to $g^{\circ}(x ; u)$ for every $u \in X$. (See Michel and Penot [7].)

It is known that the max function of semi-regular functions is semi-regular and that the semi-regularity condition can be used to establish strong calculus rules. We now give the following notion of a second-order directional derivative of a $C^{1,1}$ function $f$ in terms of the gradient function $\nabla f$. (See Yang and Jeyakumar [11] and Yang [12].)

Definition 1: Let $f: X \longrightarrow \mathbb{R}$ be a $C^{1,1}$ function and let $x \in X$. Then the generalised second-order directional derivative of $f$ at $x$ in the directions $(u, v) \in X \times X$, denoted by $f^{\infty}(x ; u, v)$, is defined by

$$
f^{\infty}(x ; u, v)=\sup _{z \in X} \limsup _{i 10} \frac{\langle\nabla f(x+s z+s u), v\rangle-\langle\nabla f(x+s z), v\rangle}{s}
$$

The generalised Hessian of $f$ at $x \in X$ for each $u \in X$, denoted by $\partial^{\infty} f(x)(u)$, is defined by

$$
\partial^{\infty} f(x)(u)=\left\{x^{*} \in X^{*}: f^{\infty}(x ; u, v) \geqslant\left\langle x^{*}, v\right\rangle, \forall v \in X\right\}
$$

The following proposition summarises some basic properties of the generalised second-order directional derivative and the generalised Hessian which are used in the sequel (see [11]). 
PROPOSITION 1. Let $f: X \longrightarrow \mathbb{R}$ be $C^{1,1}$ and $x, u, v \in X$. Then the following properties hold

(i) $f^{\infty}(x ; u, v)$ is finite and bi-sublinear as a function of $u$ and $v$;

(ii) $\partial^{\infty} f(x)(u)$ is a nonempty, convex and weak ${ }^{*}$-compact subset of $X^{*}$;

(iii) $(-f)^{\infty}(x ; u, v)=f^{\infty}(x ;-u, v)=f^{\infty}(x ; u,-v)$;

(iv) $f^{\infty}(x ; u, \alpha v)=f^{\infty}(x ; \alpha u, v), \quad \forall \alpha \in \mathbb{R} \backslash\{0\}$.

The function $f$ is said to be twice weakly Gâteaux differentiable at $x[1]$ if $f$ is continuously Gâteaux differentiable near $x$ and its gradient function $\nabla f$ is weakly Gâteaux differentiable at $x$, that is, there exists a linear function $D^{2} f(x): X \longrightarrow X^{*}$ such that for each $v \in X^{* *}, u \in X$, the following holds:

$$
\lim _{s \rightarrow 0} \frac{\langle\nabla f(x+s u), v\rangle-\langle\nabla f(x), v\rangle}{s}=\left\langle D^{2} f(x)(u), v\right\rangle .
$$

Examples of $C^{1,1}$ functions appear, for example, in penalty function methods, augmented Lagrangian methods, proximal point methods and smooth approximation methods. We now give some examples of $C^{1,1}$ functions.

EXAmple 1. Let $X=\mathbb{R}$ and let $g: \mathbb{R} \longrightarrow \mathbb{R}$ be a locally Lipschitz function. Then the function $f: \mathbb{R} \longrightarrow \mathbb{R}$, defined by

$$
f(x)=\int_{0}^{x} g(t) d t, \quad x \in \mathbb{R},
$$

is a $C^{1,1}$ function. If in addition $g$ is increasing, then $f$ is a convex $C^{1,1}$ function.

EXAMPLE 2. Let $X$ be a Hilbert space and let

$$
h(x)=\frac{1}{2}\|x\|^{2}, \quad x \in X .
$$

Then $h$ is $C^{1,1}$. Furthermore, it is twice weakly Gâteaux differentiable. We have

$$
h^{\infty}(x ; u, v)=\langle u, v\rangle, \quad \forall u, v \in X
$$

Exampie 3. Let $C$ be a subset of $X$. Define the following functions, for each $x \in X$,

$$
\begin{aligned}
d_{C}(x) & =\inf \{\|x-y\|: y \in C\}, \\
\phi(x) & =\frac{1}{2} d_{C}^{2}(x), \\
P_{C}(x) & =\left\{y \in C:\|x-y\|=\inf _{x \in C}\|x-z\|\right\} .
\end{aligned}
$$


Two special cases:

(i) $C=\{0\}$, we have $\phi(x)=1 / 2\|x\|^{2}$ which was considered in Example 2;

(ii) $C=E_{i}$, a closed interval in $\mathbb{R}$ (bounded or unbounded), then $d_{E_{i}}^{2}(x)$ can be used in formulating exterior point methods and augmented Lagrangian methods, see [9]. In particular, if $C=(-\infty, 0]$, then $\phi(x)=$ $1 / 2[\max \{x, 0\}]^{2}$.

If $C$ is a closed convex subset of a Hilbert space, then $P_{C}(\cdot)$ is single-valued, Lipschitz with Lipschitz constant $L\left(P_{C}(\cdot)\right)=1$ and

$$
\nabla \phi(\cdot)=\left(I-P_{C}\right)(\cdot)
$$

see Holmes [5]. Hence $\phi(x)$ is a $C^{1,1}$ function. The generalised second-order directional derivative of $\phi(x)$ was calculated in [12] under certain regularity conditions. We now obtain an estimate of the generalised second-order directional derivative for this function without regularity conditions.

Proposition 2. Let $X$ be a Hilbert space. If $C$ is a closed convex subset of $X$, then

$$
\phi^{\infty}(x ; u, u) \leqslant 0, \quad \forall u \in X
$$

ProOF: Since $P_{C}$ is Lipschitz with Lipschitz constant $L\left(P_{C}(\cdot)\right)=1$ (see Example 3), we have from (4)

$$
\begin{aligned}
\left(d_{C}^{2}\right)^{\infty} & (x ; u, u) \\
& =\sup _{z \in X} \limsup _{s \downarrow 0} \frac{\left\langle 2\left(P_{C}-I\right)(x+s u+s z), u\right\rangle-\left\langle 2\left(P_{C}-I\right)(x+s z), u\right\rangle}{s} \\
& =\sup _{z \in X} \limsup _{s \downarrow 0} \frac{2\left\langle P_{C}(x+s u+s z)-P_{C}(x+s z),-u\right\rangle-2 s\langle u, u\rangle}{s} \\
& =2 \sup _{z \in X} \limsup _{s \downarrow 0} \frac{\left\langle P_{C}(x+s u+s z)-P_{C}(x+s z),-u\right\rangle}{s}-2\langle u, u\rangle \\
& \leqslant 0, \quad \forall x, u \in X .
\end{aligned}
$$

Then (5) holds.

\section{Max Function and Generalised Hessian}

In this section, we study generalised differentiability properties of the max functions of the form

$$
m_{p}(x)=[\max \{g(x), 0\}]^{p}, \quad x \in X
$$


where $X$ is a Banach space, $g: X \rightarrow \mathbb{R}$ and $p \geqslant 2$. It is known that the max function $m_{p}(x)$ is (Gâteaux) differentiable if $g$ is (Gâteaux) differentiable. Indeed, we have

$$
\nabla m_{p}(x)=p[\max \{g(x), 0\}]^{p-1} \nabla g(x), \quad \forall x \in X .
$$

When $g$ has twice differentiability properties and $p=2$, various generalised Hessians of the function $m_{2}$ have been obtained, for example, in $[2,4,11,14]$. We are now able to obtain a characterisation of the generalised Hessian of $m_{p}$ in terms of the generalised Hessians of $g$ when $g$ is $C^{1,1}$ function. Moreover, we obtain necessary and sufficient conditions for $m_{p}$ to be twice weakly Gateaux differentiable.

THEOREM 1. Let $g: X \longrightarrow \mathbb{R}$ be $C^{1,1}$ and $p \geqslant 2$. Then $m_{p}(x)=[\max \{g(x), 0\}]^{p}$ is $C^{1,1}$ and for each $u \in X$, the generalised second-order directional derivative of $m_{p}$ at $x$ is given by

$$
m_{p}^{\infty}(x ; u, v)= \begin{cases}p g(x) g^{\infty}(x ; u, v)+p\langle\nabla g(x), u\rangle\langle\nabla g(x), v\rangle, & \text { if } g(x)>0 \\ 0, & \text { if } g(x)<0 \\ p \max \{\langle\nabla g(x), u\rangle\langle\nabla g(x), v\rangle, 0\}, & \text { if } g(x)=0 .\end{cases}
$$

Proof: Since $g$ is $C^{1,1}$, it is clear from (7) that $m_{p}$ is $C^{1,1}$. For simplicity, we prove the results for the case $p=2$. We shall consider the following three cases:

CASE I. Let $g(x)>0$.Then we have from (7) that the equality, $\nabla f(x)=2 g(x) \nabla g(x)$, holds in a neighbourhood of $x$. Since $g$ is $C^{1,1}$, it is semi-regular and so, we get

$$
\begin{aligned}
m_{2}^{\infty}(x ; u, v) & \\
= & \sup _{z \in X} \limsup _{s \downarrow 0} \frac{1}{s}\{2 g(x+s u+s z)\langle\nabla g(x+s u+s z), v\rangle \\
& \quad-2 g(x+s z)\langle\nabla g(x+s z), v\rangle\} \\
= & \sup _{z \in X} \limsup _{s \downarrow 0} \frac{1}{s}\{2 g(x+s z)(\langle\nabla g(x+s u+s z)-\nabla g(x+s z), v\rangle) \\
& \quad+2(g(x+s u+s z)-g(x+s z))\langle\nabla g(x+s u+s z), v\rangle\} \\
= & \sup _{z \in X} \limsup _{s \downarrow 0} \frac{1}{s}\{2 g(x)(\langle\nabla g(x+s u+s z)-\nabla g(x+s z), v\rangle) \\
& \quad+2(g(x+s u+s z)-g(x+s z))\langle\nabla g(x), v\rangle\} \\
= & \sup _{z \in X} \limsup _{s \downarrow 0} \frac{1}{s} 2 g(x)(\langle\nabla g(x+s u+s z)-\nabla g(x+s z), v\rangle) \\
& \quad+\lim _{s \downarrow 0} \frac{1}{s} 2(g(x+s u)-g(x))\langle\nabla g(x), v\rangle \\
= & 2 g(x) g^{\infty}(x ; u, v)+2\langle\nabla g(x), u\rangle\langle\nabla g(x), v\rangle,
\end{aligned}
$$

thus the result holds. 
CASE II. Let $g(x)<0$. Then we obtain $m_{2}(x)=0$ in a neighbourhood of $x$. Hence the result is true.

CASE III. Let $g(x)=0$. In fact, when $p=2$, (7) becomes

$$
\nabla m_{2}(x)=2 \max \{g(x), 0\} \nabla g(x), \quad \forall x \in X
$$

For each $z \in X$, we get

$$
\lim _{s \downarrow 0} \frac{\max \{g(x+s z), 0\}(\langle\nabla g(x+s u+s z), v\rangle-\langle\nabla g(x+s z), v\rangle)}{s}=0 .
$$

Thus we have

$$
\begin{aligned}
m_{2}^{\infty}(x ; u, v) & \\
= & \sup _{z \in X} \limsup _{s 10} \frac{1}{s}\{2 \max \{g(x+s u+s z), 0\}\langle\nabla g(x+s u+s z), v\rangle \\
& \quad-2 \max \{g(x+s z), 0\}\langle\nabla g(x+s z), v\rangle\} \\
= & \sup _{z \in X} \limsup _{s \downarrow 0} \frac{1}{s}[2 \max \{g(x+s z), 0\}(\langle\nabla g(x+s u+s z), v\rangle-\langle\nabla g(x+s z), v\rangle) \\
& \quad+2(\max \{g(x+s u+s z), 0\}-\max \{g(x+s z), 0\})\langle\nabla g(x+s u+s z), v\rangle] \\
= & \sup _{z \in X} \limsup _{\bullet \downarrow} \frac{2(\max \{g(x+s u+s z), 0\}-\max \{g(x+s z), 0\})\langle\nabla g(x), v\rangle}{s} .
\end{aligned}
$$

Since $g$ is $C^{1,1}, \max \{g, 0\}$ is semi-regular, thus we obtain

$$
\begin{aligned}
& m_{2}^{\infty}(x ; u, v) \\
& \quad=2 \lim _{\cdot 10} \frac{\max \{g(x+s u)\langle\nabla g(x), v\rangle, 0\}-\max \{g(x)\langle\nabla g(x), v\rangle, 0\}}{s} \\
& \quad=2 \max \{\langle\nabla g(x), u\rangle\langle\nabla g(x), v\rangle, 0\} .
\end{aligned}
$$

Then the proof is complete.

REMARK 1. From the Hahn-Banach Theorem [5], we get the following inclusions of the generalised Hessian,

$$
\begin{aligned}
\partial^{\infty} m_{p}(x)(u) & \\
= & \left\{\begin{array}{rr}
\left\{p g(x)^{p-1} x+p(p-1) g(x)^{p-2}\langle\nabla g(x), u\rangle \nabla g(x):\right. & \\
\left.x^{*} \in \partial^{\infty} g(x)(u)\right\}, & \text { if } g(x)>0 \\
\{0\}, & \text { if } g(x)<0 \\
\left\{\beta p(p-1) g(x)^{p-2}\langle\nabla g(x), u\rangle \nabla g(x): \beta \in[0,1]\right\}, & \text { if } g(x)=0
\end{array}\right.
\end{aligned}
$$


REMARK 2. It follows from a second-order chain rule (see [12, Theorem 2]) that

$$
\begin{aligned}
& \partial^{\infty} m_{p}(x)(u) \\
& \subseteq\left\{\begin{array}{rr}
\left\{p g(x)^{p-1} x+p(p-1) g(x)^{p-2}\langle\nabla g(x), u\rangle \nabla g(x):\right. & \\
\left.x^{*} \in \partial^{\infty} g(x)(u)\right\}, & \text { if } g(x)>0 ; \\
\{0\}, & \text { if } g(x)<0 ; \\
\left\{\beta p(p-1) g(x)^{p-2}\langle\nabla g(x), u) \nabla g(x): \beta \in[0,1]\right\}, & \text { if } g(x)=0 .
\end{array}\right.
\end{aligned}
$$

and that (9) holds with equality if $\nabla g(x)$ is onto. Comparing (8) with (9), we see that the onto condition used in $[11]$ is only sufficient.

Using Theorem 1, we obtain characterisations of twice weakly Gâteaux differentiability of the max function $m_{p}$ when the function $g$ is $C^{1,1}$.

Proposition 3. Let $X$ be a reflexive Banach space and let $g$ be $C^{1,1}$ and $x \in X$ be a point satisfying $g(x)=0$. Then the function $m_{p}$ is twice weakly Gâteaux differentiable at $x$ if and only if $\nabla g(x)=0$ and $g$ is twice weakly Gâteaux differentiable at $x$.

Proof: From Theorem 1, $\partial^{\infty} m_{p}(x)(u)$ is single-valued for all $u \in X$ if and only if $\nabla g(x)=0$ and $\partial^{\circ} g(x)(u)$ is single-valued for all $u \in X$. Then the conclusion holds.

We finish this section with a couple of numerical examples to show the structure of the generalised Hessian of max functions.

EXAmple 4. Let $m_{p}(x)=[\max \{x, 0\}]^{p}, x \in \mathbb{R}$ and $p \geqslant 2$. Then we have

$$
\partial^{\infty} m_{p}(x)(u)= \begin{cases}\left\{p(p-1) x^{p-2} u\right\}, & \text { if } x>0 \\ \{0\}, & \text { if } x<0 \\ \left\{\beta p(p-1) x^{p-2} u: \beta \in[0,1]\right\}, & \text { if } x=0 .\end{cases}
$$

EXAMPLE 5. Let $m_{2}(x)=\left[\max \left\{\int_{0}^{x} t^{2} \sin (1 / t) d t+1,0\right\}\right]^{2}, x \in \mathbb{R}$. Then our generalised Hessian $\partial^{\infty} m_{2}(x)(u)$ at $x=0$ is

$$
\partial^{\infty} m_{2}(0)(u)=\{0\} .
$$

\section{Weak Convexity and Generalised Second-Order Derivative}

In this section, we obtain a characterisation of $\eta$-convexity and show that every $C^{1,1}$ function is locally weakly convex in a Hilbert space using the generalised secondorder directional derivative $f^{\infty}(x ; u, v)$.

We first recall the definition of $\boldsymbol{\eta}$-convexity. 
Definition 2. Let $C$ be a convex subset of $X$ and let $f: C \longrightarrow \mathbb{R}$. The function $f$ is said to be $\eta$-convex on $C$ if there exist a real number $\eta$ and a convex function $h: C \longrightarrow \mathbb{R}$ such that $f(x)=h(x)+\eta\|x\|^{2}, \forall x \in C$.

Note that if $\eta>0$, then $f$ is said to be strongly convex on $C$; if $\eta=0$, then $f$ is convex on $C$; if $\eta<0$, then $f$ is said to be weakly convex on $C$, see Vial [10] and Jeyakumar [6].

DEFINITION 3. (i) $f: X \longrightarrow \mathbb{R}$ is said to be locally weakly convex on $X$ if for each $x \in X$, there exists $r>0$ such that $f$ is weakly convex on an open ball centred at $x$ with radius $r$, denoted by $U^{\circ}(x, r)$;

(ii) $f$ is said to be globally weakly convex if $f$ is weakly convex on $X$.

The following characterisation for a $C^{1,1}$ function to be convex is given in [11].

LEMma 1. Let $X$ be a Banach space and let $f: X \longrightarrow \mathbb{R}$. Then $f$ is convex on $X$ if and only if

$$
f^{\infty}(x ; u,-u) \geqslant 0, \quad \forall x, u \in X
$$

We first obtain a characterisation of $\eta$-convexity in terms of the generalised secondorder directional derivative. It is worth noting that this result paves the way to establishing and generalising connections between a $C^{1,1}$ function and weak convexity in a Hilbert space.

Theorem 2. Let $X$ be a Hilbert space and let $f: X \longrightarrow \mathbb{R}$ be $C^{1,1}$. Then $f$ is $\eta$-convex on $X$ if and only if

$$
f^{\infty}(x ; u,-u) \geqslant-2 \eta\|u\|^{2}, \quad \forall x, u \in X
$$

ProOF: Let $f$ be a $C^{1,1}$ function. If $f$ is $\eta$-convex on $X$, then there exist a real number $\eta$ and a convex function $h: X \longrightarrow \mathbb{R}$ such that $f(x)=h(x)+\eta\|x\|^{2}, \forall x \in$ $X$. Since $f$ and $\eta\|\cdot\|^{2}$ are $C^{1,1}$, the function $h$ is also $C^{1,1}$. Note from (3) that $\left(\|\cdot\|^{2}\right)^{\infty}(x ; u,-u)=-2\|u\|, \forall u \in X$. Hence from the triangle inequality, we obtain

$$
\begin{aligned}
f^{\infty} \cdot(x ; u,-u) & \leqslant h^{\infty}(x ; u,-u)+\left(\eta\|\cdot\|^{2}\right)^{\infty}(x ; u,-u) \\
& \leqslant h^{\infty}(x ; u,-u)-2 \eta\|u\|^{2}, \quad \forall x, u \in X .
\end{aligned}
$$

From Lemma $1, h^{\infty}(x ; u,-u) \leqslant 0, \forall x, u \in X$, so we have

$$
f^{\infty}(x ; u,-u) \leqslant-2 \eta\|u\|^{2}, \quad \forall x, u \in X .
$$

Conversely, if (10) holds, then

$$
f(x)=\left(f(x)-\eta\|x\|^{2}\right)+\eta\|x\|^{2}, \quad \forall x, u \in X,
$$


and the function $f(x)-\eta\|x\|^{2}$ is convex on $X$ since

$$
\left(f-\eta\|\cdot\|^{2}\right)^{\infty}(x ; u,-u) \leqslant f^{\infty}(x ; u,-u)+2 \eta\|u\|^{2} \leqslant 0, \quad \forall x, u \in X .
$$

Thus $f$ is $\eta$-convex on $X$.

Clearly Theorem 2 is an extension of Lemma 1 . Moreover, when $\eta=0$, Theorem 2 reduces to Lemma 1 . As an immediate application of Theorem 2 , let $g: \mathbb{R} \longrightarrow \mathbb{R}$ be a locally Lipschitz function. Then the function $f$ defined in Example 1 is $\eta$-convex if and only if $g^{\diamond}(x ;-1) \leqslant 2 \eta, \forall x \in \mathbb{R}$. The following corollary shows that Theorem 2 generalises a result in [10, Proposition 4.11] where twice differentiability is required.

Corollary 1. Let $X$ be a Hilbert space and let $f: X \rightarrow \mathbb{R}$ be twice weakly Gâteaux differentiable. Then $f$ is $\eta$-convex on $X$ if and only if

$$
\left\langle D^{2} f(x)(u), u\right\rangle \geqslant 2 \eta\|u\|^{2}, \quad \forall x, u \in X .
$$

Proof: This follows from the fact that $f$ is twice weakly Gâteaux differentiable, thus

$$
f^{\infty}(x ; u,-u)=-\left\langle D^{2} f(x)(u), u\right\rangle, \forall x, u \in X .
$$

Now we establish that in a Hilbert space every $C^{1,1}$ function is locally weakly convex using our generalised second-order directional derivative.

Theorem 3. Let $X$ be a Hilbert space. If $f: X \longrightarrow \mathbb{R}$ is a $C^{1,1}$ function, then $f$ is locally weakly convex on $X$.

ProOf: Let $f: X \longrightarrow \mathbb{R}$ be a $C^{1,1}$ function. Then for any fixed $\bar{x} \in X$, it follows from the locally Lipschitz condition of $\nabla f$ that there exist $L(\nabla f, \bar{x})>0$ and $r>0$ such that

$$
\|\nabla f(y)-\nabla f(x)\| \leqslant L(\nabla f, \bar{x})\|y-x\|, \quad \forall y, x \in U^{\circ}(\bar{x}, r) .
$$

Let $\eta \geqslant(L(\nabla f, \bar{x})) / 2$. Then for any $u \in X, x \in U^{\circ}(\bar{x}, r)$, we have

$$
\begin{aligned}
f^{\infty}(x ; u,-u) & =\sup _{z \in X} \limsup _{s 10} \frac{\langle\nabla f(x+s u+s z),-u\rangle-\langle\nabla f(x+s z),-u\rangle}{s} \\
& \leqslant L(\nabla f, \bar{x})\|u\|^{2} \leqslant 2 \eta\|u\|^{2} .
\end{aligned}
$$

So,

$$
\begin{aligned}
\left(f+\eta\|\cdot\|^{2}\right)^{\infty}(x ; u,-u) & \leqslant f^{\infty}(x ; u,-u)+\left(\eta\|\cdot\|^{2}\right)^{\infty}(x ; u,-u) \\
& =f^{\infty}(x ; u,-u)-2 \eta\|u\|^{2} \\
& \leqslant 0, \quad \forall x \in U^{\circ}(\bar{x}, r), u \in X
\end{aligned}
$$


From Lemma $1, f+\eta\|\cdot\|^{2}$ is convex on $U^{\circ}(\bar{x}, r)$. Then $f(x)=\left(f(x)+\eta\|x\|^{2}\right)-\eta\|x\|^{2}$, in which $f+\eta\|\cdot\|^{2}$ is convex on $U^{\circ}(\bar{x}, r)$. Hence $f$ is locally weakly convex on $X$.

It is well known that the function $-d_{C}^{2}(x)$ is globally weakly convex, where $C$ is a closed convex subset of a Hilbert space. We present a proof of this result using our generalised second-order directional derivative. Recall that $-d_{C}^{2}(x)$ is a $C^{1,1}$ function, see Example 3.

Proposition 4. Let $X$ be a Hilbert space. If $C$ is a closed convex subset of $X$, then $-d_{C}^{2}(x)$ is globally weakly convex.

Proof: Observe that

$$
-d_{C}^{2}(x)=\left(2\|x\|^{2}-d_{C}^{2}(x)\right)-2\|x\|^{2} .
$$

Thus we need to prove that $x \longrightarrow 2\|x\|^{2}-d_{C}^{2}(x)$ is convex on $X$. From Proposition 2 , we have

$$
\left(-d_{C}^{2}\right)^{\infty}(x ; u,-u)=\left(d_{C}^{2}\right)^{\infty}(x ; u, u) \leqslant 0, \quad \forall x, u \in X
$$

Then from (3)

$$
\begin{aligned}
\left(2\|\cdot\|^{2}-d_{C}^{2}\right)^{\infty}(x ; u,-u) & \leqslant\left(2\|\cdot\|^{2}\right)^{\infty}(x ; u,-u)+\left(-d_{C}^{2}\right)^{\infty}(x ; u,-u) \\
& \leqslant-4\langle u, u\rangle \\
& \leqslant 0, \quad \forall x, u \in X .
\end{aligned}
$$

From Lemma 1, the function $x \longrightarrow 2\|x\|^{2}-d_{C}^{2}(x)$ is convex on $X$. Therefore $-d_{C}^{2}(x)$ is globally weakly convex.

Corollary 2. Let $X$ be a Hilbert space and let $g: X \rightarrow \mathbb{R}$ be a convex function. Then $m_{2}(x)=-[\max \{g(x), 0\}]^{2}$ is globally weakly convex.

Proof: Let $C=\{x \in X: g(x) \leqslant 0\}$. Thus $C$ is a closed convex subset and $d_{C}^{2}(x)=[\max \{g(x), 0\}]^{2}$. The conclusion follows from Proposition 4.

\section{DISCUSSION}

Let $X$ be a Hilbert space and let $f: X \longrightarrow \mathbb{R}$. Then the following classes of functions are introduced and studied in $[3,6,8,10]$ :

(i) the function $f$ is said to be locally difference convex on $X$ if for every $\bar{x} \in X$, there exist a convex neighbourhood $N(\bar{x})$ of $\bar{x}$, and convex functions $p_{N}, q_{N}: X \rightarrow \mathbb{R}$ such that $f(x)=p_{N}(x)-q_{N}(x), \forall x \in N(\bar{x})$. This class of functions is denoted by $\mathrm{LDC}(\mathrm{X})$. The function $f$ is said to be difference convex on $X$ if there exist two convex functions $p, q: X \longrightarrow \mathbb{R}$ such that $f(x)=p(x)-q(x), \forall x \in X$; 
(ii) the function $f$ is said to be lower- $C^{2}$ on $X$ if for every $\bar{x} \in X$, there exist a convex neighbourhood $N(\bar{x})$ of $\bar{x}$, a convex function $p_{N}$ and a quadratic convex function $q_{N}$ such that $f(x)=p_{N}(x)-q_{N}(x), \forall x \in N(\bar{x})$. This class of functions is denoted by $L C^{2}(X)$.

It follows from the previous definitions that every locally weakly convex function is locally difference convex. In general, a quadratic convex function in a Hilbert space has the form

$$
\langle A(u), u\rangle+\langle b, u\rangle+c,
$$

where $b \in X, c \in \mathbb{R}$ and $A: X \longrightarrow X$ satisfies $\langle A(x), x\rangle \geqslant 0,\langle A(x), y\rangle=\langle A(y), x\rangle$. In particular $\|x\|^{2}=\langle x, x\rangle$ is a quadratic convex function. Hence it follows from Theorem 3 that every $C^{1,1}$ function is lower- $C^{2}$. It is clear that every lower- $C^{2}$ function is locally difference convex. Therefore we have established that

$$
C^{1,1}(X) \subset L C^{2}(X) \subset L D C(X),
$$

where $X$ is a Hilbert space. This result was initially given in Hiriart-Urruty [3] and Vial [10] in a finite dimensional space.

\section{REFERENCES}

[1] J.P. Aubin and I. Ekeland, Applied nonlinear analysis (John Wiley, New York, 1984).

[2] A. Auslender, 'Penalty methods for computing points that satisfy second order necessary conditions', Math. Programming 17 (1979), 229-238.

[3] J.B. Hiriart-Urruty, 'Generalized differentiability, duality and optimization for problems dealing with difference of convex functions', in Convexity and duality in optimization, ( $\mathrm{J}$. Ponstein, Editor), Lecture Notes in Economics and Mathematical Systems, 256, 1984, pp. 37-70.

[4] J.B. Hiriart-Urruty, J.J. Strodiot and V. Hien Nguyen, 'Generalized Hessian matrix and second-order optimality conditions for problems with $C^{1,1}$ data', Appl. Math. Optim. 11 (1984), 43-56.

[5] R.B. Holmes, Geometric functional analysis and its applications (Springer-Verlag, Berlin, Heidelberg, New York, 1975).

[6] V. Jeyakumar, 'On subgradient duality with strong and weak convex functions', J. Austral. Math. Soc. Ser. A 40 (1986), 143-152.

[7] P. Michel and J.P. Penot, 'A generalized derivative for calm and stable functions', Differential Integral Equations 5 (1991), 433-454.

[8] R.T. Rockafellar, 'Favorable classes of Lipschitz-continuous functions in subgradient optimization', in Progress in Nondifferentiable Optimization, (E.A. Nurminski, Editor) (Internat. Inst. Appl. Systems. Anal., Laxenburg, 1982), pp. 125-143.

[9] R.T. Rockafellar, 'First- and second-order epi-differentiability in nonlinear programming', Trans. Amer. Math. Soc. 307 (1988), 75-108. 
[10] J.P. Vial, 'Strong and weak convexity of sets and functions', Math. Oper. Res. 8 (1983), 231-259.

[11] X.Q. Yang and V. Jeyakumar, 'Generalized second-order directional derivatives and optimization with $C^{1,1}$ functions', Optimization 26 (1992), 165-185.

[12] X.Q. Yang, 'Generalized second-order directional derivatives and optimality conditions', Nonlinear Anal. 22 (1994), 767-784.

[13] X.Q. Yang, 'Smoothing approximations to nonsmooth optimization problems', J. Austral. Math. Soc. Ser. B 36 (1994), 1-13.

[14] X.Q. Yang, 'An exterior point method for computing points that satisfy second-order necessary conditions for a $C^{1,1}$ optimization problem', J. Math. Anal. Appl. 187 (1994), 118-133.

Department of Mathematics

The University of Western Australia

Nedlands, QA 6009 Australia 